\title{
Gel Stent Implantation-Recommendations for Preoperative Assessment, Surgical Technique, and Postoperative Management
}

\author{
Vanessa Vera, ${ }^{1}$ Iqbal "Ike" K Ahmed, ${ }^{2}$ Ingeborg Stalmans, ${ }^{3}$ and Herbert Reitsamer ${ }^{4}$ \\ 1. Department of Ophthalmology, Unidad Oftalmológica de Caracas, Venezuela; 2. Department of Ophthalmology and Vision Sciences, University \\ of Toronto, Ontario, Canada; 3. Department of Ophthalmology, University Hospitals Leuven (UZLeuven), Belgium; 4. Department of Ophthalmology, \\ Paracelsus University Salzburg, Austria
}

DOl: https://doi.org/10.17925/USOR.2018.11.1.38

$\mathrm{T}$ he surgical management of glaucoma offers the potential to lower intraocular pressure (IOP) independent of patients' compliance with their medication regimen. Procedures such as trabeculectomy and tube shunt placement often yield large magnitudes of IOP reduction, but may be associated with short- and long-term complications. Microinvasive glaucoma surgery (MIGS) offers an alternative surgical approach that is inherently less invasive; however, most devices that fit in this category are associated with a lesser degree of IOP-lowering efficacy compared with traditional glaucoma surgeries. A newer MIGS device, a gel stent that facilitates drainage to the subconjunctival space, appears to offer similar IOP reduction to trabeculectomy, but with much less tissue manipulation; better predictability; and less sight-threatening complications, thus making it a potentially safer and more predictable surgical option in appropriate patients. The following proposed protocol, based on evidence-based practices and augmented where necessary by the opinions of experienced surgeons, provides guidance for the pre-, intra-, and postoperative management of patients receiving a gel stent implant. The goal of this protocol is to provide a framework for better patient selection and preparation, surgical pearls, and how best to assess and manage patients in the postoperative period.

\section{Keywords}

Glaucoma, microinvasive glaucoma surgery (MIGS), gel stent, gel implant

Disclosure: Vanessa Vera has served as a consultant to Allergan, Aquesys, and KeloTec. Iqbal "Ike" K Ahmed has served as a consultant to Acucela, Aerie Pharmaceuticals, Alcon, Allergan, ArcScan, Bausch \& Lomb, Carl Zeiss Meditec, Centervue, Clarity Medical Systems, ElutiMed, Envisia Therapeutics, Eyelight, Equinox, ForSight Labs, Glaukos, Gore, lantech, InjectSense, InnFocus, Iridex, iStar, Ivantis, Johnson \& Johnson Vision, KeLoTec, LayerBio, Leica Microsystems, New World Medical, Omega Ophthalmics, Ono Pharma, PolyActiva, Sanoculis, Santen, Science Based Health, SOLX, Stroma, and TrueVision; Research grant from Aerie Pharmaceuticals, Alcon, Allergan, Carl Zeiss Meditec, Glaukos, Ivantis, Johnson \& Johnson Vision, New World Medical; Speaker for Alcon, Allergan, Carl Zeiss Meditec, Johnson \& Johnson Vision. Ingeborg Stalmans has served as a consultant to Aerie, Alcon, Allergan, EyeTechCare, Santen, Théa. Herbert Reitsamer has served as a consultant to Alcon, Aliophta, Allergan, Askin, Aquesys, Bausch \& Lomb, Bayer, Carl Zeiss Meditec, CellSeed Inc., Chibret, Chroma Pharma, Heidelberg Engineering, Hoya, Implandata Ophthalmic Products, Ivantis, Johnson \& Johnson Vision, KeloTec, MSD, Novartis, Perimed Polytech Domilens, Rayner, Santen, Schwind Eye Solutions, Théa, and Transcend.

Acknowledgments: Medical writing assistance was provided by Bryan Bechtel of Bechtel Editorial Services, LLC. He has no disclosures to report.

Compliance with Ethics: This article provides evidence-based expert recommendations and did not involve any studies with human or animal subjects performed by any of the authors.

Authorship: All named authors meet the International Committee of Medical Journal Editors (ICMJE) criteria for authorship of this manuscript, take responsibility for the integrity of the work as a whole, and have given final approval to the version to be published. All authors contributed equally to this manuscript.

Open Access: This article is published under the Creative Commons Attribution Noncommercial License, which permits any noncommercial use, distribution, adaptation, and reproduction provided the original author(s) and source are given appropriate credit. (C) The Authors 2018.

Received: September 18, 2017

Accepted: October 30, 2017

Citation: US Ophthalmic Review, 2018;11(1):38-46

Corresponding Author: Vanessa Vera, 28372 Via Pasito. San Juan Capistano, CA, 92675. E: vanessaveramd@gmail.com

Support: The publication of this article was supported by Allergan.
Trabeculectomy and tube shunt surgeries have an important role in glaucoma management, offering the ability to achieve intraocular pressure (IOP) lowering independent of patients' compliance in appropriate candidates. Both trabeculectomy and tube shunt drainage devices, performed using an ab externo approach, achieve lowering of IOP via drainage of aqueous from the anterior chamber (AC) to the subconjunctival space. ${ }^{1}$ However, each of these procedures is associated with a lengthy and variable recuperation and prolonged time to visual recovery. In some instances, there is associated loss of best-corrected visual acuity (BCVA) and significant risk of short- and long-term complications, including hypotony, choroidal effusion, cataract, and flat or shallow anterior chamber, as well as valve-related complications, such as tube blockage, erosion, and endothelial cell loss. ${ }^{1-4}$

An alternative option, in the form of microinvasive glaucoma surgery (MIGS) devices, is associated with clinically relevant IOP lowering with an improved safety profile and recovery compared with trabeculectomy and tube shunts. ${ }^{5}$ There are several devices fitting the definition of MIGS; they can be categorized by their intended mechanism of action. ${ }^{6}$ For instance, they may improve outflow by targeting the trabecular meshwork (TM), Schlemm canal, or the supraciliary space.

A MIGS drainage device implanted via an ab interno approach that facilitates drainage of aqueous from the $\mathrm{AC}$ to the subconjunctival space (Xen ${ }^{\circledR} 45$; Allergan, Irvine, CA) has recently been approved in the US for use in the surgical management of glaucoma. This device, which can be used as a standalone procedure, offers the potential to achieve IOP lowering that is comparable to trabeculectomy, but with less tissue manipulation.7.8 The device, which is implanted using a handheld disposable injector, is 
constructed from porcine-derived gelatin that is formed into a tube and cross-linked with glutaraldehyde, thus allowing it to expand and remain flexible when hydrated. The expansion of the tube's outer diameter when in contact with fluid also aids in retention of the gel stent in its intended location after surgical implantation, which helps minimize many of the issues associated with synthetic materials, such as migration, erosion, and corneal endothelial damage. ${ }^{9}$ The gel stent was designed using principles established by the Hagen-Poiseuille equation, whereby outflow resistance to the fluid increases linearly in proportion to the length of the tube, and decreases to the fourth power of the diameter of the tube lumen. In sum, a longer and thinner tube provides greater resistance than a shorter and wider tube. ${ }^{10}$

In the US, the gel stent is indicated for the management of refractory glaucomas, including cases where previous surgical treatment has failed, and cases of primary open-angle glaucoma, pseudoexfoliative, or pigmentary glaucoma with open angles that are unresponsive to maximum tolerated medical therapy. The device has been given the Conformité Européene (CE) mark for use in Europe, where it is indicated for the reduction of IOP in patients with primary open-angle glaucoma where previous medical treatments have failed. It is also licensed for use in Canada and Turkey, and has recently gained marketing approval in select countries in Latin America and Asia. To date, more than 24,000 gel stent devices have been implanted worldwide. ${ }^{11}$

\section{Expert principles for implantation}

The following recommendations were developed based on the clinical experience of the authors, all of whom were involved in the development of the gel stent and also have considerable surgical experience with the device. The objective of the protocol is to present evidence-based practices, augmented with the opinion of the authors, as guidance for the management of gel stent patients during the pre-, intra-, and postoperative periods. (Note: for the purposes of this paper, the terms "gel implant" and "gel stent" will be used interchangeably.)

A summary of this protocol is available in Figure 1. Fundamentally, the guidance is informed by three main principles: to minimize bleeding during implantation, lower conjunctival resistance, and control inflammation. Bleeding during implantation may impair visibility of crucial steps of the implant procedure and/or incite the release of inflammatory cytokines ${ }^{12}$ that facilitate fibrosis. ${ }^{13,14}$ It has been reported that lowering conjunctival resistance to outflow increases the likelihood of achieving sustained IOP lowering. ${ }^{13}$ Finally, control of inflammation mediates an important cause of fibrosis and bleb failure in subconjunctival filtering procedures. ${ }^{13,14}$

\section{Preoperative considerations}

Although not explicitly stated as part of the "preparation" phase noted in Figure 1, proper patient selection and preparation are implied critical elements for successful gel stent implantation. Also falling under the category of "preparation" is education and counseling of patients so as to establish reasonable postoperative expectations.

Implantation of the gel stent has been shown to correspond to an early postoperative pressure gradient between 6 and $8 \mathrm{mmHg}$ following an uncomplicated implantation under normal physiologic conditions and without conjunctival resistance to the outflow. ${ }^{10}$ The pressure gradient is a result of the resistance of the implant (which stays constant) and the
Figure 1: XEN-EP (Expert Principles)

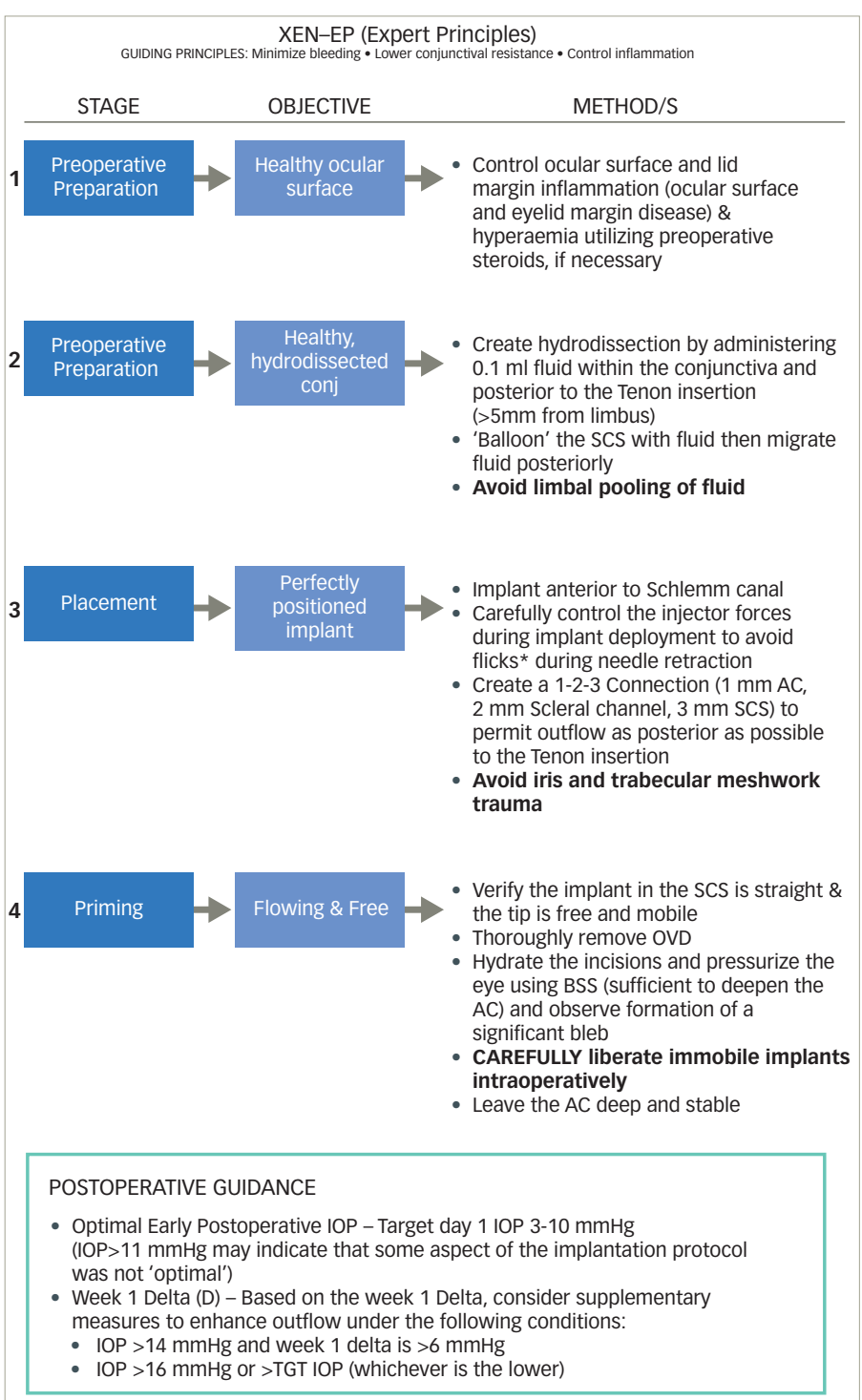

$A C=$ anterior chamber; $B S S=$ balanced saline solution; $O V D=$ ophthalmic viscosurgical device; SCS = subconjunctival space. *Flicks are an unintended vertical or horizontal bend force on the needle sleeve. When not recognized and not corrected, they may result in an up, side, or downward "flick" of the sleeve when the needle retracts out of the scleral tissue. This may result in damage to the stent or to the tissue.

resistance to outflow under the conjunctiva (which changes as the bleb evolves over time). Thus, as general guidance, patients requiring a target pressure in the low to mid-teens would seem to be suitable candidates for this type of implant. Implantation of the gel stent represents a minimally invasive and more controlled and predictable means of achieving a pressure within this range while also reducing (and in many cases eliminating) the need for postoperative IOP-lowering medication use. As a result, postoperative recovery is often faster compared with trabeculectomy and tube surgeries. ${ }^{7,15}$

Detecting and treating comorbid conditions, particularly ocular surface disease/dry eye and blepharitis, can help reduce important causes of inflammation. ${ }^{16}$ In the presence of conjunctival injection, stopping IOP-lowering medications (and, if necessary, replacing them with oral acetazolamide) a few weeks prior to surgery may be considered along with use of topical steroids and preservative-free lubricants to reduce ocular 
Figure 2: Preoperative protocol

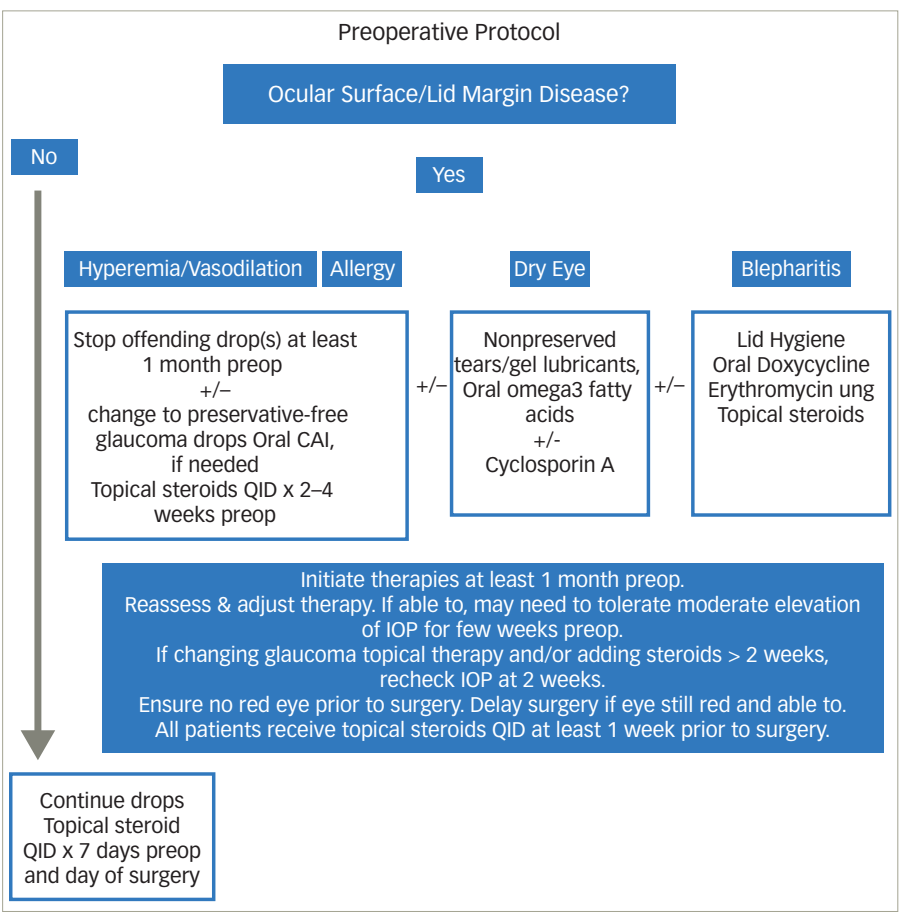

$C A I=$ carbonic anhydrase inhibitors; IOP = intraocular pressure; $Q I D=$ four times a day; ung $=$ ointment

surface inflammation (Figure 2). ${ }^{17}$ Operating on a quiet eye with a healthy conjunctiva, and preferably one that has not undergone previous surgery involving conjunctiva in the target quadrant, establishes conditions that increase the likelihood of successful implantation. Pseudophakic eyes, or those planned to undergo concomitant cataract surgery, may comprise a more straightforward operating environment early in the surgeon's learning curve, allowing easier access (i.e. a deep AC) and accurate implant placement at the angle, with less potential to induce inflammatory responses. Also, the concomitant need for cataract surgery may represent an opportunity to simultaneously perform a procedure that will help the patient achieve control of IOP along with visual rehabilitation.

\section{Perioperative considerations}

The "preparation" phase of gel stent implantation continues to the perioperative period with choice of anesthesia, which will likely depend on several factors; however, pain avoidance will decrease the potential for release of proinflammatory cytokines, ${ }^{18}$ and attendant scar tissue formation and bleb failure. ${ }^{14}$ Surgeons may opt to use a regional block, especially in early cases, to minimize the potential for patient or ocular movements that may lead to iatrogenic trauma. Generally speaking, the anesthesia requirement for implantation of the gel stent is no different to that of other ocular surgeries, including trabeculectomy. Many surgeons find the use of topical anesthesia alone to be more than sufficient for surgical comfort.

\section{Hydroexpansion and adjunctive use of antifibrotic agents during gel stent surgery}

The objective of hydroexpansion is to separate conjunctival and Tenon tissue layers surrounding and adjacent to the desired implant location in the subconjunctival space, ${ }^{13}$ thus preparing the space for the implant and supporting formation of the bleb (see step 2, Figure 1). Performing this step may have the added benefit of reducing Tenon tissue resistance in the immediate postoperative period to improve flow.

Hydroexpansion is achieved via introduction of $0.1 \mathrm{~mL}$ of fluid at least $5 \mathrm{~mm}$ from the limbus, preferably using a 30-gauge needle (see Mitomycin C [MMC] Injection Technique Video, available online at www.touchOPHTHALMOLOGY. com). There is supporting evidence from studies on trabeculectomy that the use of antifibrotic agents inhibit multiplication of cells that produce scar tissue, thus facilitating healing of the conjunctiva and formation of a bleb..$^{19}$ The dosage used and mechanism of delivery of the antifibrotic agent may also be important for the final outcome. Pakravan et al. reported that sub-Tenon injection of $0.1 \mathrm{~mL}$ of $0.01 \% \mathrm{MMC}$ was more likely to result in a favorable bleb appearance (i.e. more diffuse, shallow, and normally vascularized) compared with use of cellulose sponges soaked with $0.02 \%$ $\mathrm{MMC}$ that were placed under the Tenon tissue and on the conjunctiva over the scleral flap for 1 or 3 minutes in eyes undergoing trabeculectomy. ${ }^{20}$

There is growing evidence showing a similar benefit in gel implant surgeries. Galal et al. reported on a protocol using sub-Tenon injection of $0.1 \mathrm{~mL} \mathrm{MMC} 0.01 \%$ (equivalent to an absolute dose of $10 \mu \mathrm{g}$ ) that was spread with a microsponge on the conjunctiva in the target quadrant 10 minutes prior to gel stent implantation. ${ }^{21}$ De Gregorio et al. reported use of $0.1 \mathrm{~mL}$ of $\mathrm{MMC}$ in a concentration of $0.1 \mathrm{mg} / \mathrm{mL}$ (absolute dose of $10 \mu \mathrm{g}$ ) injected subconjunctivally in the superotemporal quadrant and then rolled superonasally prior to gel stent surgery in standalone procedures and in surgeries combined with phacoemulsification..$^{15} \mathrm{~A}$ large retrospective study comparing outcomes with gel stent and trabeculectomy by Schlenker et al. reported perioperative use of MMC injection in standalone and combined surgeries using a concentration of $0.2 \mathrm{mg} / \mathrm{mL}$ in volumes ranging from $0.05-0.2 \mathrm{~mL}$ (equivalent to an absolute dose of 10-40 $\mu \mathrm{g}$ ). ${ }^{?}$

In the context of trabeculectomy, use of lower doses of MMC is associated with adequate suppression of fibrosis, with a better safety profile compared with higher doses. ${ }^{22}$ As such, the authors' techniques for using MMC have evolved over the course of their experience implanting the gel stent, moving over time to lower doses than are typically used in trabeculectomy surgeries. In the authors' opinion, injecting a large volume of $\mathrm{MMC}$, over $0.1 \mathrm{~mL}$, increases the risk of pooling in the limbus, anterior bleb formation, and conjunctival avascularity. Based on the above-mentioned literature, ,15,22 the authors typically use MMC at a concentration of 0.1 to $0.2 \mathrm{mg} / \mathrm{mL}$ (most often in a total volume of $0.1 \mathrm{~mL}$; absolute dose range of 10-20 $\mu \mathrm{g}$ ), which is a lower concentration than what is often used during trabeculectomy. In eyes that demonstrate a propensity for scarring, a concentration of up to $0.4 \mathrm{mg} / \mathrm{mL}$ (at total volume of $0.1 \mathrm{~mL}$; absolute dose of up to $40 \mu \mathrm{g}$ ) may be required. However, there may be a need to adjust the dose based on the needs of the patient, and the authors recommend caution with use of higher concentrations of MMC.23

Placement of the hydroexpansion agent intra-Tenon (rather than subconjunctival), with location as far posterior to the limbus as possible, helps to maintain the applied antifibrotic in the desired quadrant. While not published in peer-reviewed literature, some of the authors previously preferred injection in the temporal quadrant followed by use of a cottontipped swab to move the fluid to the desired location. This preference was later abandoned due to concerns that temporal injection may induce conjunctival changes in the area, thereby complicating future surgery should it be necessary. Instead, the authors suggest injection at 
or around the $12 \mathrm{o}^{\prime}$ clock position, aiming slightly superonasally $>5 \mathrm{~mm}$ posterior to the limbus at the intended location of the gel implant. This placement will allow the hydroexpansion fluid to migrate without pooling at the limbus. To avoid bleeding, blood vessels should be avoided as the hydroexpansion needle is introduced into and through the subconjunctival space. Bleeding may compromise visibility or result in bleb malfunction postoperatively. ${ }^{4}$

\section{Surgical technique}

Achieving a perfectly positioned implant must engender consideration for optimal positioning with respect to quadrant, which, in turn, has a bearing on where the bleb will form. Because a nasally forming bleb may result in bleb dysesthesia, cosmetic issues, and/or bleb-related complications, ${ }^{24}$ ideal gel stent positioning would appear to be between 12:00 and 1:30 o'clock OD and between 10:30 to 12:00 o'clock OS. While positioning as close to 12:00 $o^{\prime}$ clock as possible is preferable, this level of precision may be difficult to achieve, especially during the learning curve. Accurate positioning may also be difficult in patients with either a deep eye or prominent cheekbone, in which case a more temporal incision with a more tangential pass will permit easier access for the injector needle.

During all phases of implanting the gel stent, it is critical to avoid iatrogenic trauma to reduce the risk of bleeding events. Conjunctival bleeding events, if they occur, can typically be addressed using external tamponade with a blunt instrument.

Before undertaking the principle steps of the surgery, reference markings can be placed $3.0 \mathrm{~mm}$ from the limbus at the desired implant location. The primary incision should be made $90-180^{\circ}$ from the intended stent location, with a sideport incision of approximately $1.0 \mathrm{~mm}$ in length. Next, a cohesive viscoelastic is used to fill the AC.

Following preparation of the injector, the tip is inserted through the primary incision and the needle is advanced through the AC. Gonioscopy is helpful in guiding the injector to the target location in the angle, although some of the authors do not use this visualization technique at this stage of the operation (see XEN Implantation No Gonio for Angle Entry Video available at www.touchOPHTHALMOLOGY.com). The objective is to insert the tip of the needle just anterior to the pigmented TM through nonpigmented TM and just below the Schwalbe line (see XEN Implantation Gonio Assisted Video, available at www.touchOPHTHALMOLOGY.com). This will ensure that the final implant position will be anterior to the canal of Schlemm, thereby avoiding blood reflux into the AC that results from needle passage through the Schlemm canal. Without gonioscopy, the perception of depth and the position of the needle can be misleading. Consequently, surgeons might have the impression of being more anterior than they actually are. To counter this tendency, it may be helpful to bring the tip of the injector to the presumed area of the TM and then to lift the tip slightly to achieve the desired placement.

A second instrument is inserted into the sideport (e.g. a Vera Fixation Hook [Asico, LLC; Westmont, IL]) and the needle is then advanced through the sclera. The entire bevel of the needle should emerge about 3.0 $\pm 0.5 \mathrm{~mm}$ from the limbus, near the conjunctival markings if they were placed at the beginning of the surgery. Once the bevel of the needle is fully visible, some surgeons aim to achieve a more superficial placement under the conjunctiva, whereas others aim for a deeper placement. It is unclear if superficial versus deeper positioning is better, and it is the authors' experience that a completely free and mobile implant at the end of the procedure, which is the ultimate goal, can be achieved with either placement.

After the desired position placement of the needle bevel is accomplished, the surgeon should then gently and slowly slide the injector plunger until full deployment is achieved. Care should be taken during retraction of the needle to avoid a flick, an unintended vertical or horizontal bending of the needle sleeve; if a flick is not recognized and corrected, it could result in an abrupt movement of the sleeve when the needle retracts out of the scleral tissue, resulting in damage to the stent or surrounding tissue. To avoid these flicks, release tension on the injector and side instrument, keep a gentle forward bias to avoid retraction of the sleeve from the angle during the injection phase, focus attention on the limbus (rather than the needle tip), and only retract the injector when the slider is completely forward.

At this point in the surgery, the surgeon should use gonioscopy to ensure that around $1.0 \mathrm{~mm}$ of the implant projects into the angle and that placement is immediately anterior to the pigmented TM.

\section{Confirming final placement and outflow}

The "priming" phase of gel stent implantation begins with an inspection of the implant location. Following the surgery, the implant should be observable as a pale yellow tube through the intact conjunctiva. The mnemonic "1-2-3" may be used as a guide for achieving optimum final implant positioning, referring to the implant extending about $1.0 \mathrm{~mm}$ into the anterior chamber at its proximal end, $2.0 \mathrm{~mm}$ of the implant in the scleral tunnel, and $3.0 \mathrm{~mm}$ at the distal end of the implant lying flat in the subconjunctival space. This positioning will (1) reduce the risk of implant occlusion in the anterior chamber; (2) avoid implant migration; and (3) permit optimum bleb formation and outflow as posterior to the limbus as possible.

At the end of the implant procedure, the gel stent should be free and mobile. It should be straight and not bent or curled. A curled implant may be caused by a number of potential mechanisms, including tissue interference as the implant enters the subconjunctival space during implantation. Other potential causes include incomplete exposure of the injector needle bevel resulting in incomplete dissection of the scleral tissue that restricts passage of the implant during deployment; a soft/hydrated implant due to prior entry into the eye, for example, where previous attempts to implant the gel stent have been made (thereby exposing the implant to moisture); incomplete or inadequate hydroexpansion of the conjunctival tissue space prior to implantation; and if the implant's scleral exit point coincides with the point of Tenon layer insertion (where the tissue density/resistance may be particularly high). Gentle manipulation of the implant tip through the intact conjunctiva will ensure it is free and mobile and that outflow is not restricted. If positioning is not satisfactory (i.e. bent more than $90^{\circ}$ and/ or immobile), further manipulation via needle revision might be necessary (discussed in greater detail below).

Viscoleastic should be thoroughly rinsed from the eye intraoperatively to reduce the risk for stent blockage and elevated postoperative IOP. Following this step, constant irrigation of balanced salt solution (BSS) into the AC is needed to prime the implant and induce formation of a bleb (see Bleb Priming Video, available at www.touchOPHTHALMOLOGY.com). If subconjunctival 
Figure 3: Postoperative management recommendations-Day 1 to Week 1

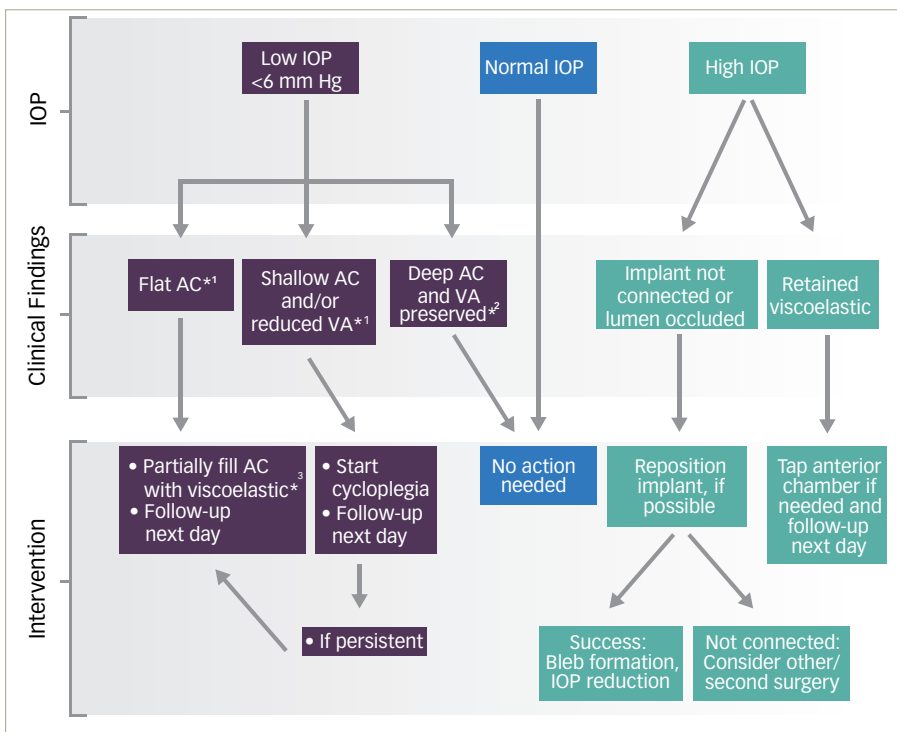

*1 With or without choroidal detachment; ${ }^{* 2}$ No choroidal detachment; ${ }^{* 3}$ AVOID high molecular weight viscoelastic. $A C=$ anterior chamber; $I O P=$ intraocular pressure; VA = visual acuity

placement of the gel implant was intended and successfully achieved, a diffuse (not localized) bleb should form rapidly, with dispersion of liquid under the conjunctiva appearing like a "water wave" over the sclera.

If present, any and all blood should be thoroughly removed from the AC as this is a risk factor for implant blockage and release of inflammatory cytokines $^{12}$ that may increase the likelihood of postoperative bleb failure..$^{13,14}$ The final part of the "priming" phase is to thoroughly hydrate all incisions to ensure that pressure and a formed AC are maintained.

\section{Considerations for unsatisfactory implant placements}

Following implantation, if the gel stent is deemed immobile or excessively curled or twisted, the surgeon should consider liberating the implant intraoperatively (the authors refer to this as step as "primary needling"). The technique for achieving this, while analogous to the "needling" procedure described in the literature ${ }^{10}$ used to mechanically break down scar tissue following failed glaucoma filtration procedures, is much less invasive. One approach to performing this maneuver is use a gentle "slide and swipe" technique by inserting a 30-gauge needle below the implant and then to moving it in the direction of the fornix, repeating this with insertion of the needle above the implant when necessary (see Primary Needling Video, available at www.touchOPHTHALMOLOGY.com).

Primary needling is the authors' preferred first option for an immobile implant when the implant is excessively curled (i.e. "pig tailed"), which is unlikely to provide for adequate posterior aqueous flow postoperatively, or when the implant is placed such that interstitial resistance could limit aqueous flow. If the implant is free and mobile at its distal end, primary needling may not be necessary even if the implant is slightly curved. Nevertheless, the surgeon may still wish to correct the implant's positioning using a blunt instrument, applying gentle posterior force and directing it away from the limbus. If unsuccessful with this step, manipulation of the conjunctiva around the area of the implant may free the obstruction.
As a last resort, primary needling of the surrounding tissue may liberate the implant with the caveat that this step may be associated with increased risk of bleeding.

As noted earlier, a 1-2-3 positioning is most likely to achieve the desired location of outflow postoperatively. If that is not achieved, the implant should be repositioned before the surgery is concluded. The authors' opinions for adjusting suboptimal implant length position is as follows.

- Not connected or too short in the AC: in scenarios where the connection into the $\mathrm{AC}$ is deemed too short or not visible (i.e. implant is buried into angle tissue), a reasonable step is to use forceps at the scleral exit site, pushing the implant back slightly into the AC.

- Not connected or too short in subconjunctival space: if the implant is too short under the conjunctiva or not visible, the most straightforward solution is usually to remove the implant from the AC through the main incision with microforceps (i.e. vitrectomy forceps, anterior chamber microinstruments, etc.), reload the implant in the injector, and attempt a second implantation maneuver. This approach is also recommended when the implant is placed too nasally or if the scleral channel is too short.

- Slightly short in subconjunctival space: in scenarios where small length adjustments are necessary under the conjunctiva, it is reasonable to use forceps to pull the implant further into the subconjunctival space. It is important to understand that because of the conjunctival anatomy and insertion at the limbal area, a tissue slack will be necessary before grabbing, holding, and pulling the implant out into the subconjunctival space (see Repositioning video, available at www.touchOPHTHALMOLOGY.com).

\section{Postoperative considerations}

Similar to trabeculectomy, the requirements for the postoperative period following gel stent implantation are based on IOP measurements taken during follow-up examination (Figures 3 and 4). However, there are important differences to note between the surgeries with respect to change in pressure over time and the bleb morphology and function. Proper preparation, placement, and priming of a gel stent implant should result in a low-lying and diffuse bleb that is morphologically different than what is typically observed following trabeculectomy (Figure 5). Additionally, the internal resistance of the gel stent is designed to prevent acute decompression in the early postoperative period.

\section{Optimal intraocular pressure in the early postoperative period}

Postoperative management of patients following gel stent implantation is guided by the philosophy of "start low, stay low" with respect to IOP. A postoperative IOP between 3 and $10 \mathrm{mmHg}$ is ideal for the immediate postoperative days with this device, and any deviation outside this norm may indicate inadequate outflow performance. However, a successful outcome is still achievable with postoperative pressure readings above or below this range. Related to this last point, it is important to differentiate between cases in which very low IOP meets the technical definition of hypotony (also referred to as numerical hypotony) from those in which hypotony manifests clinical consequences, such as hypotony maculopathy (i.e. clinical or symptomatic hypotony). Based on the authors' experiences, an IOP $\leq 6 \mathrm{mmHg}$ in the immediate postoperative period with the gel stent seems less likely to lead to 


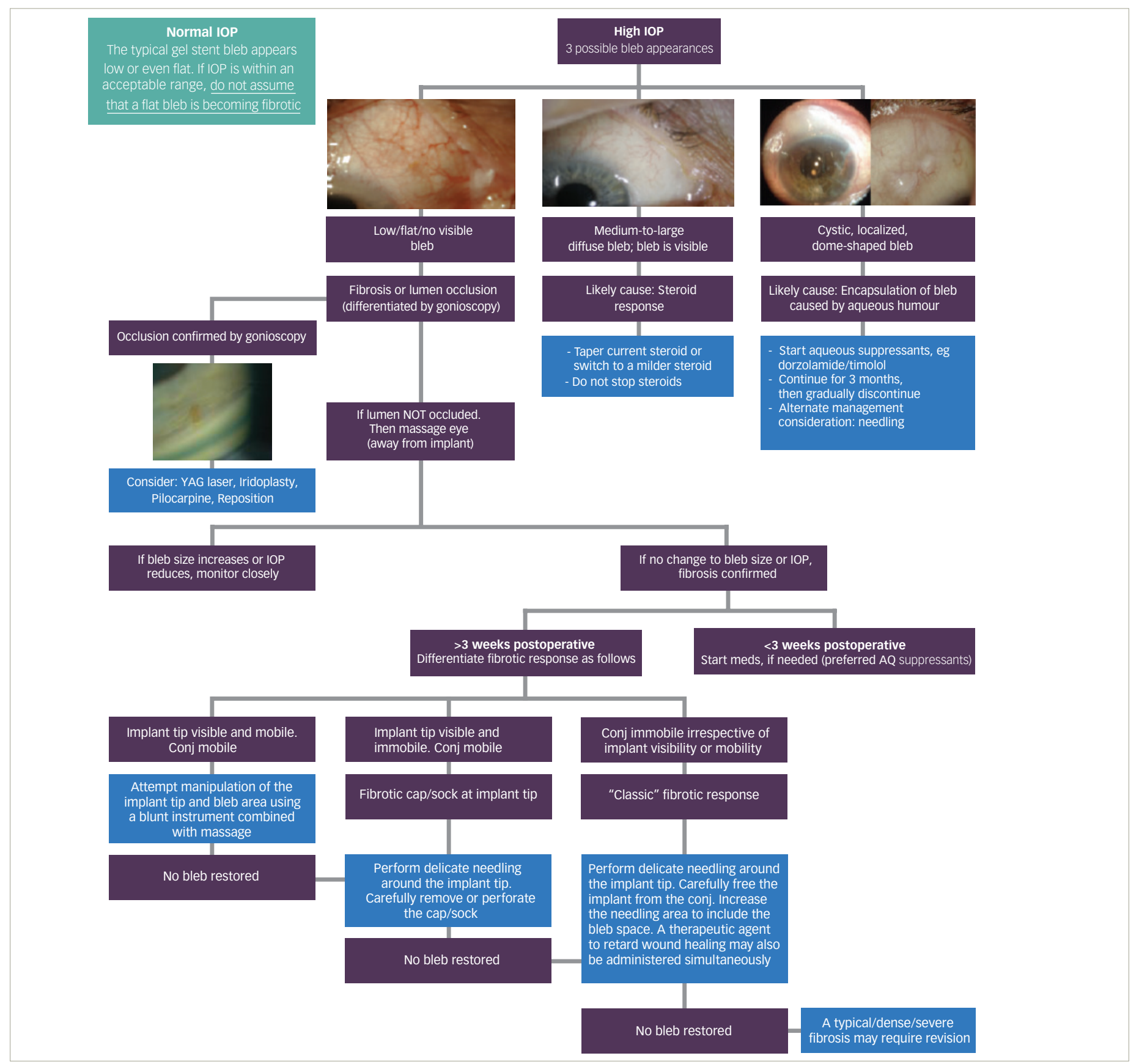

${ }^{*}$ Assuming implant is confirmed to be connected. $1 O P=$ intraocular pressure.

clinical hypotony-related complications compared to similar IOP after trabeculectomy, and such cases after gel stent implantation may be amenable to observation without immediate intervention.

There are several factors that may lead to the development of hypotony following gel stent implantation. For example, impaired aqueous production rates (e.g. in patients with uveitis); wound leak; and eye rubbing. Intraoperative risks, such as cyclodialysis resulting from misplacement of the injector needle or traumatic "flick" action of the injector during implantation (for example, traumatic ciliary body shutdown, clefts, etc.), or a requirement for multiple passes with the injector needle through the TM, may also contribute to lower than expected IOP. Other potential risks that may lead to hypotony include over-filtration around the tube (side or para flow) - such as may occur secondary to scleral hysteresis, use of a short scleral channel, or in an eye with high myopia and/or thin sclera-and use of aqueous suppressant medication prior to surgery.

\section{Low intraocular pressure (clinically significant hypotony)} In instances where clinically significant hypotony is suspected, the evaluation and management should be differentiated based on the likely or suspected cause, with extra attention given to those cases in which there is accompanying loss of visual acuity. In exceptional situations where 
Table 1: Management of Elevated IOP (>15 mmHg) within 1 month postoperative*

\begin{tabular}{|c|c|c|c|c|c|}
\hline & $\begin{array}{l}\text { Blocked Lumen/ } \\
\text { Not Connected }\end{array}$ & Fibrotic Bleb & $\begin{array}{l}\text { High Interstitial } \\
\text { Resistance Bleb }\end{array}$ & Steroid Response & Encapsulated Bleb \\
\hline IOP & Very elevated & Slowly increasing & Early mild elevation & Mild to moderate elevation & [Very] elevated \\
\hline Bleb & Flat & Flattening & Diffuse, quiet & Diffuse, large, quiet & Dome shaped, quiet \\
\hline $\begin{array}{l}\text { Additional } \\
\text { Features }\end{array}$ & $\begin{array}{l}\text { Internal lumen blocked } \\
\text { (iris, blood, pigment, OVD) } \\
\text { Nonvisible AC/SCS }\end{array}$ & $\begin{array}{l}\text { Vessel dilation and } \\
\text { tortuosity }\end{array}$ & $\begin{array}{l}\text { reduced visibility and/or } \\
\text { mobility, increased curling? }\end{array}$ & $\begin{array}{l}\text { Excellent early IOP and } \\
\text { bleb, IOP elevated despite } \\
\text { ideal bleb }\end{array}$ & Defined, very elevated bleb \\
\hline Recommendation & $\begin{array}{l}\text { Release iris } \\
\text { DOC to clear lumen } \\
\text { AC tap if OVD } \\
\text { Implant repositioning } \\
\text { Revision }\end{array}$ & $\begin{array}{l}++ \text { Steroids } \\
\text { Anti-metabolite injection- } \\
\text { Needling } \\
\text { DOC }\end{array}$ & $\begin{array}{l}\text { DOC } \\
\text { Needling }\end{array}$ & Weaker/taper steroids & $\begin{array}{l}\text { Aqueous suppressants } \\
\text { Needling } \\
\text { Revision }\end{array}$ \\
\hline
\end{tabular}

*This table builds on recommendations provided in Figures 3 and 4. AC = anterior chamber; DOC = digital ocular compression; IOP = intraocular pressure; OVD = ophthalmic viscosurgical device; $S C S=$ subconjunctival space.

viscoelastic materials are needed to increase the viscosity of aqueous in patients with symptomatic hypotony, thereby increasing resistance to outflow, high-molecular-weight cohesive viscoelastic should not be used and, instead, low-molecular-weight dispersive viscoelastic should be used to partially fill the AC (Figure 2). Discretion should be used regarding the volume of viscoelastic used. Alternatively, the use of air, either alone or with viscoelastic, can be considered.

\section{Elevated intraocular pressure}

IOP measurements above the target range may not be indicative of implant failure, but care should be taken to find the cause and attempt to fix it if necessary (Table 1). Confirming proper implant connection to the AC and subconjunctival space should always be the first step in the evaluation. After confirming implant connection, other plausible mechanisms for postoperative IOP above the target range include retained viscoelastic, blockade of the implant lumen in the $\mathrm{AC}$, fibrosis, bleb encapsulation, and impaired bleb function, similar to what may be encountered after trabeculectomy and tube surgeries. ${ }^{25,26}$

The ostium of the implant can be blocked by a number of mechanisms, including, but not limited to, iris occlusion, lens fragment material (in the case of combined surgeries), and blood. Several risk factors have been identified to increase the risk of iris occlusion with tube shunts that may apply to implantation of a gel stent, including intraoperative floppy iris syndrome (IFIS), peripheral iris abnormality, low positioning into the angle/iris root (Figure 6), narrow angle, phakic lens status, and previous iridotomy in the vicinity of the implantation (owing to the risk of iris strands incarcerating the internal ostium). ${ }^{27,28}$ Iris occlusion of the gel stent implant is a rarely occurring event when proper implant placement is achieved.

If any form of obstruction is suspected, clinical factors, such as elevated IOP (with the magnitude of elevation proportional to the level of occlusion), absent or low bleb, and patient complaints of transient discomfort with IOP spikes occurring at nighttime or under low-light conditions, can be used to determine the need for intervention. The following options may be considered for iris blockage: $532 \mathrm{~nm}$-laser iridoplasty, adjust the implant length in the AC (by gently pulling or pushing the implant further into the $\mathrm{AC}$ ), administration of a miotic agent (a trial a few days prior to any intervention may help determine whether removing the obstruction is achievable), or nd:YAG laser. If laser is to be used, it is the authors' opinion that a local $532 \mathrm{~nm}$-laser laser iridoplasty may be preferable, while a full iridotomy should be avoided due to risk of releasing additional iris material into the $\mathrm{AC}$, elevating the risk of inflammation. ${ }^{29}$

Elevated IOP occurring after 1 week likely indicates a different set of mechanisms that necessitate different corrective measures. For example, outflow restriction causing elevation of IOP should be evaluated on the basis of whether the implant is mobile and visible. When the implant is mobile and visible in the subconjunctiva, the objective is to overcome tissue resistance around the implant, first via digital ocular compression (DOC; also referred to as ocular massage), followed by needle revision of the bleb if massage fails. This maneuver is performed by applying manual pressure on the globe through the lid in the inferonasal region for about 10 seconds, depressing the eye backward and slightly up. A visible but immobile implant may indicate fibrosis requiring needling at the distal end of the implant; however, care should be taken not to cut or break the implant. The needling action should be directed below and above the implant in order to remove any tissue obstruction, fibrotic cap, or plug that may have formed in the region, keeping in mind that this step may be associated with increased risk of hemorrhage, bleb leakage, and implant breakage (See Needling a Failed Xen Video, available at www.touchOPHTHALMOLOGY.com). ${ }^{14}$ Instances in which the implant is not visible under the conjunctiva indicate a need for a more aggressive intervention, up to and including surgical revision.

On the other hand, less commonly, ${ }^{30}$ when encapsulation occurs, the use of preservative-free aqueous suppressant, including oral acetazolamide if needed, with reevaluation at a later date, is a reasonable first approach (Figure 7). In some cases of encapsulation, needling may be considered, although there is a risk of recurrence. It is the authors' opinion that a flat bleb with elevated IOP in the early postoperative period is most likely due to fibrosis, high interstitial resistance, or implant blockage, whereas restriction of flow due to encapsulation of the bleb is a much more likely cause of a dome-shaped or elevated bleb, especially if it occurs 1 month postoperatively. The response to DOC may help determine if the bleb is functional, which, in turn, will dictate the course of additional intervention. 
Figure 5: Slit-lamp images 1 year after gel stent implantation OU

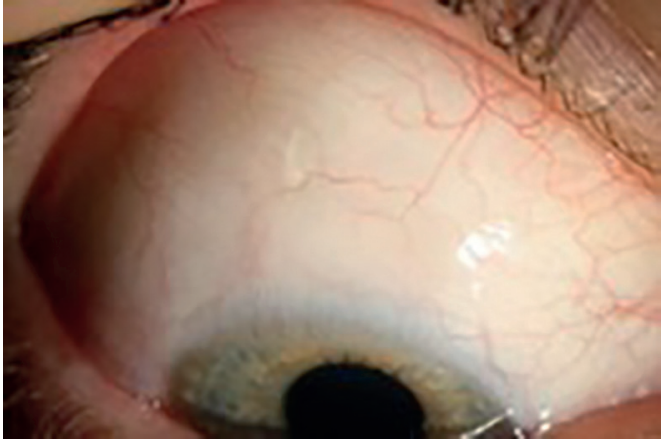

B

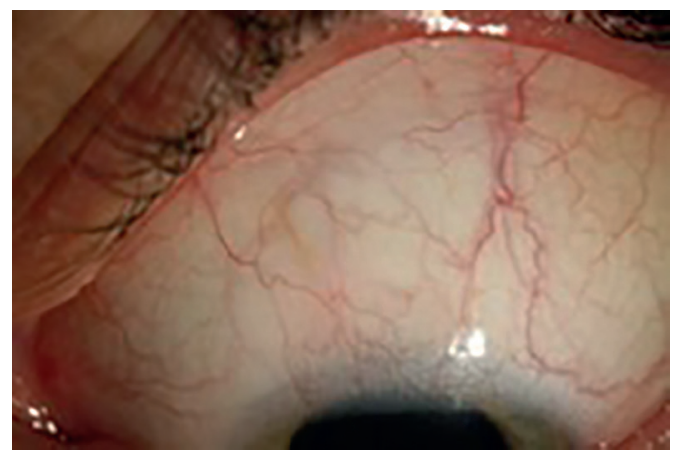

A 36-year-old patient with primary open-angle glaucoma; preoperative IOP of $28 \mathrm{mmHg}$ OD and $26 \mathrm{mmHg}$ OS on 3 medications OU. Images show low-lying diffuse bleb ou 1 year after implantation: IOP is $13 \mathrm{mmHg}$ without medications $O D(A) ; I O P$ is $13 \mathrm{mmHg}$ on 3 medications OS (B). Courtesy of Ingeborg Stalmans, MD

Figure 6: Gonioview of implant blocked by iris at 2 weeks after gel stent implantation (intraocular pressure $21 \mathrm{mmHg}$ ) in a phakic eye

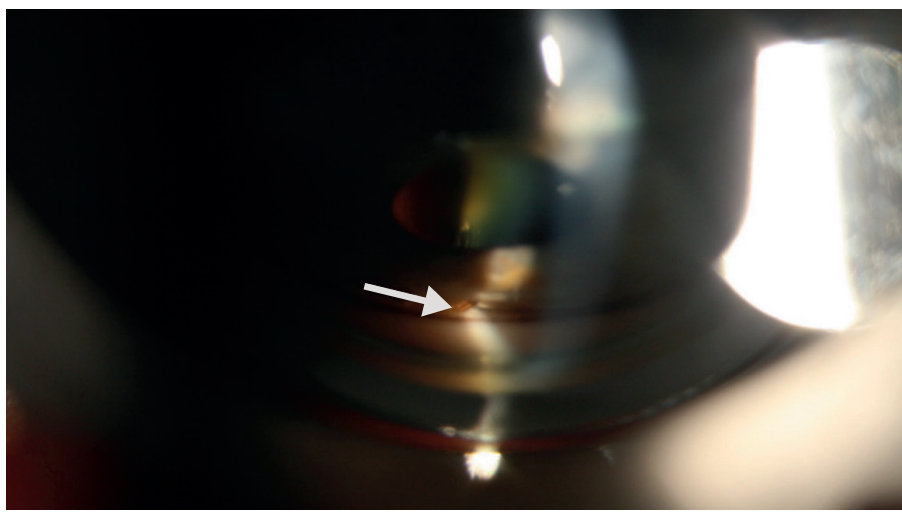

Note the low implant entry placement (under trabecular meshwork). Courtesy of Ingeborg Stalmans, MD.

The potential role of corticosteroids in inducing IOP elevation must be ruled out before determining if a corrective measure is indicated prior to any intervention.

\section{The role of postoperative intraocular pressure as prognostic factor}

The proper implantation of the gel stent results in a predictable postoperative IOP, as noted earlier. Hence, any variation outside this range
Figure 7: Slit-lamp image of right eye 4 months after receiving a gel stent implant

A

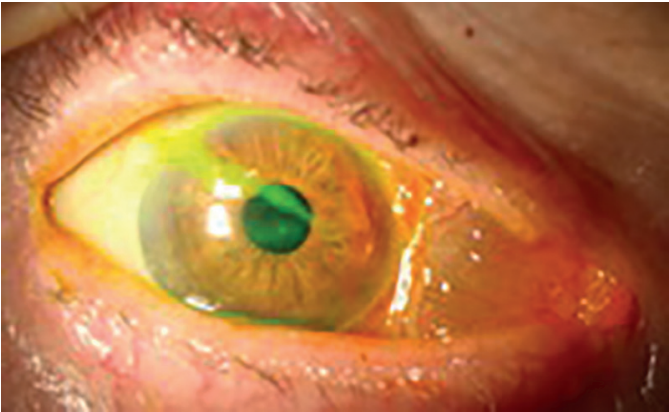

B

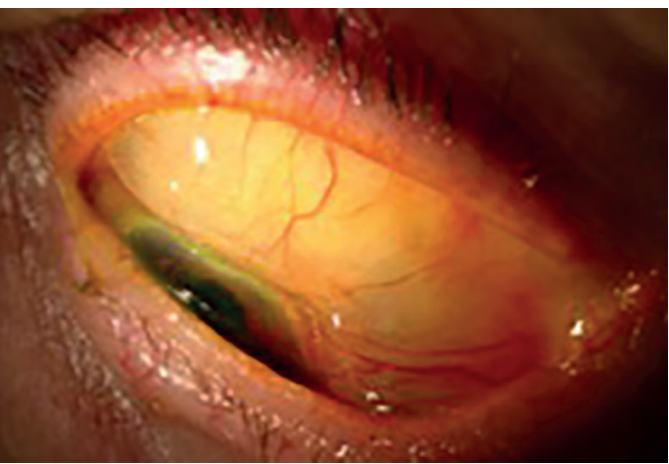

C

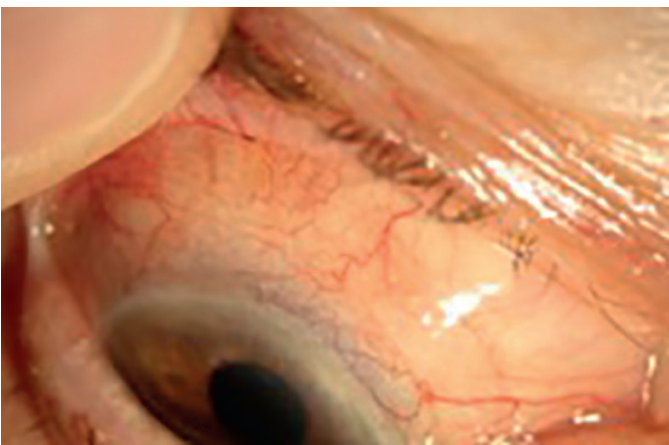

Note the unusually large encapsulated bleb. The patient had an intraocular pressure (IOP) of $26 \mathrm{mmHg}$ and complained of discomfort (A and B). One week after treatment with aqueous suppressants, the bleb was low lying and IOP was $15 \mathrm{mmHg}$ on 3 medications (C). Courtesy of Ingeborg Stalmans, MD.

can be used as a prognostic factor regarding the success of the surgery and to guide the need for further intervention (Figures 3 and 4). As noted above, when day 1 postoperative IOP is $>10 \mathrm{mmHg}$ in the presence of a confirmed connected and patent implant in the AC and subconjunctival space, the surgeon can, with some degree of confidence, deduce that the hydroexpansion component of the implantation was not performed optimally and/or that viscoelastic material was retained in the AC. The two conditions are indistinguishable based on clinical appearance, and each has separate implications for IOP recovery, thus necessitating different management strategies.

Similarly, the change of IOP at week 1 compared to day 1 (which the authors refer to as Week 1 Delta) may be used as an early prognosticator of bleb function and whether appropriate outflow has been achieved. Based on this, we propose an algorithm based on the week 1 IOP that may be useful for management of cases with "atypical" pressure measurements (Figure 4 and Table 1). It is the authors' collective experience that a significant 
increase in outflow resistance, indicated by marked increase in Week 1 Delta, may indicate a physical obstruction of the implant or increased outflow resistance (also referred to as high interstitial resistance).

We propose that an IOP measurement $\geq 16 \mathrm{mmHg}$ at Week 1 , or an IOP measurement $\geq 14 \mathrm{mmHg}$ within the first week and a Week 1 Delta $\geq 6 \mathrm{mmHg}$, should prompt investigation into likely causes. Possible causes of elevated IOP during this period may include: iris block, high interstitial resistance, and early fibrotic or encapsulation changes in the bleb. However, there are currently no studies with the gel implant investigating the role of medication at this early phase, the effect of DOC to relieve tissue resistance, or whether needle revision of the bleb is desirable as a first-line option. The authors therefore state that the choice of intervention to rectify elevated IOP should be made on a case-by-case basis with consideration given to the magnitude of IOP elevation and change in Week 1 Delta, the desire of the patient and treating physician to cease use of IOP-lowering medications use, and whether the patient is intolerant or nonadherent to medications

\section{Bleb characteristics and the role of needle revision}

Traditional clinical assessment of bleb morphology used for trabeculectomy blebs may not have prognostic value with regard to optimal flow characteristics. Instead, the surgeon should rely more on the IOP rather than solely on the appearance of bleb to guide assessment of bleb function.

Following implantation of a gel stent, the bleb will stabilize over time, with the rate of change influenced by the process of postoperative wound healing. If the desired pressure is achieved and the eye appears quiet with a low diffuse bleb, steroids can be tapered as early as 4-6 weeks after surgery. However, in eyes with active inflammation or when future interventions seem necessary, steroids should be continued. When there is restriction of flow to the bleb, there may be a need for needle revision. As with trabeculectomy, the prognosis for needle revision is better for a flatter bleb compared to cases in which the bleb is elevated, thickened, or has a cystic, dome-shaped appearance, especially if the gel stent is not visible through the bleb.

In cases where bleb needling is performed, adjunctive use of MMC or fluorouracil (5-FU) may be useful. When MMC is used, the authors use a dose that is similar to or slightly higher than what was used intraoperatively during hydroexpansion, typically in concentrations ranging from $0.1 \mathrm{mg} /$ $\mathrm{mL}$ to $0.4 \mathrm{mg} / \mathrm{mL}$ in a total volume of $0.1 \mathrm{~mL}$. It is also the authors' experience that while a successful needle revision of a bleb following gel stent implantation will immediately lower tissue resistance (defined by a significant lowering of IOP after needling), the instantaneous blebforming response is typically not as quick as that seen with needling a bleb following trabeculectomy. However, if the target IOP is not achieved, it is unlikely that needling will be successful. In general, an IOP under $10 \mathrm{mmHg}$ after needling is more likely to result in a successful outcome. Finally, use of a cohesive viscoelastic to enlarge the space around the bleb may facilitate proper bleb/space formation.

There may be a role for DOC in certain cases, either alone or following an attempted needle revision. As a standalone intervention, DOC is most helpful when the bleb is elevated but the pressure is not well controlled (for instance, if the month 1 IOP is $\geq 17 \mathrm{mmHg}$ and/or the Week 1 Delta is $\geq 6 \mathrm{mmHg}$ ), then instructing the patient to perform DOC at home could help reestablish optimal flow.

\section{Conclusions}

The logical, step-wise approach to the management of the pre-, intra-, and postoperative periods in surgeries involving the gel stent implant is intended as guidance to fellow surgeons using this MIGS device; they are meant to complement, and not to replace, formal training with the gel stent.

The Expert Principles for gel stent implantation described herein are organized into three phases: preparation, placement, and priming, with specific steps of each informed by three guiding principles-to minimize bleeding, lower conjunctival resistance, and control inflammation. While these recommendations are intended as guidance to fellow surgeons, the suggested approach may help minimize the impact of factors that could lead to a less desirable outcome. These guidelines are based on the amalgamation of evidence-based practices and the surgeon-authors' considerable experience with the gel stent implant. Just as this approach evolved over time and was enhanced by accumulation of more knowledge and experience, it is plausible that surgeons incorporating the gel stent implant into their treatment armamentarium for glaucoma will witness more favorable outcomes as they perform more cases.
1. Gedde SJ, Schiffman JC, Feuer WJ, et al., Tube versus Trabeculectomy Study Group. Treatment outcomes in the Tube Versus Trabeculectomy (TVT) study after five years of follow-up, Am J Ophthalmol, 2012;153:789-803.e2.

2. Shaarawy T, Flammer J, Haefliger IO, Reducing intraocular pressure: is surgery better than drugs?, Eye (Lond), 2004:18:1215-24.

3. Rulli E, Biagioli E, Riva I, Gambirasio G, et al., Efficacy and safety of trabeculectomy vs nonpenetrating surgical procedures: a systematic review and meta-analysis, JAMA Ophthalmol, 2013;131:1573-82.

4. Vijaya L, Manish P, Ronnie G, Shantha B, Management of complications in glaucoma surgery, Indian J Ophthalmo , 2011;59(Suppl):S131-40.

5. Saheb H, Ahmed II, Micro-invasive glaucoma surgery: current perspectives and future directions, Curr Opin Ophthalmol, 2012;23:96-104.

6. Caprioli J, Kim JH, Friedman DS, et al., Special commentary: supporting innovation for safe and effective minimally invasive glaucoma surgery: summary of a joint meeting of the American Glaucoma Society and the Food and Drug Administration, Washington, DC, February 26, 2014, Ophthalmology, 2015:122:1795-801.

7. Schlenker MB, Gulamhusein $\mathrm{H}$, Conrad-Hengerer I, et al., Efficacy, safety, and risk factors for failure of standalone ab interno gelatin microstent implantation versus standalone trabeculectomy, Ophthalmology, 2017:124:1579-88

8. Reitsamer HA, Translimbal Collage Implant. Aquesys XEN Gel Stent. In: Francis BA, Sarkisian SR, Tad JC (eds), Minimally Invasive Glaucoma Surgery, Stuttgart: Thieme, 2016.
9. Aref AA, Varma R, Advanced Glaucoma Surgery, Essentials in Ophthalmology, Switzerland: Springer, 2012

10. Sheybani A, Reitsamer H, Ahmed II, Fluid dynamics of a novel micro-fistula implant for the surgical treatment of glaucoma, Invest Ophthalmol Vis Sci, 2015;56:4789-95.

11. Allergan. Data on file.

12. Esmon $\mathrm{CT}$, The interactions between inflammation and coagulation, Br J Haematol, 2005;131:417-30.

13. Yu DY, Morgan WH, Sun $X$, et al., The critical role of the conjunctiva Yu DY, Morgan WH, Sun X, et al., The critical role of the conjunctiva
in glaucoma filtration surgery, Prog Retin Eye Res, 2009;28:303-28

14. Schlunck $G$, Meyer-ter-Vehn T, Klink T, Grehn F, Conjunctival fibrosis following filtering glaucoma surgery, Exp Eye Res, 2016;142:76-82.

15. De Gregorio A, Pedrotti E, Russo L, Morselli S, Minimally invasive combined glaucoma and cataract surgery: clinical results of the smallest ab interno gel stent, Int Ophthalmol, 2017. doi: 10.1007/ s10792-017-0571-x. [epub ahead of print].

16. Baudouin C, Ocular surface and external filtration surgery: mutual relationships, Dev Ophthalmol, 2012;50:64-78.

7. Tailor R, Batra R, Mohamed S, A national survey of glaucoma specialists on the preoperative (Trabeculectomy) management of the ocular surface, Semin Ophthalmol, 2016;31:519-25.

18. Zhang J, and An J, Cytokines, Inflammation and Pain, Int Anesthesiol Clin, 2007:45:27-37.

19. Wilkins $\mathrm{M}$, Indar A, Wormald R, Intra-operative mitomycin C for glaucoma surgery, Cochrane Database Syst Rev, 2005:CD002897

20. Pakravan M, Esfandiari H, Yazdani S, et al., Mitomycin $\mathrm{C}$-augmented trabeculectomy: sub-Tenon injection versus soaked sponges: a randomised clinical trial, Br J Ophthalmol 2017; 101:1275-80.

21. Galal A, Bilgic A, Eltanamly R, Osman A, XEN glaucoma implant with mitomycin C 1-year follow-Up: result and complications J Ophthalmol, 2017. doi: 10.1155/2017/5457246. [epub ahead of print].

22. Sanders SP, Cantor LB, Dobler AA, Hoop JS, Mitomycin C in higher risk trabeculectomy: a prospective comparison of 0.2 - to $0.4-\mathrm{mg} /$ cc doses, J Glaucoma, 1999:8:193-8.

23. Wells AP, Cordeiro MF, Bunce C, Khaw PT, Cystic bleb formation and related complications in limbus- versus fornix-based conjunctival flaps in pediatric and young adult trabeculectomy with mitomycin C, Ophthalmology, 2003;110:2192-7.

24. Budenz DL, Hoffman K, Zacchei A, Glaucoma filtering bleb dysesthesia, Am J Ophthalmol, 2001;131:626-30.

25. Bae K, Suh W, Kee C, Comparative study of encapsulated blebs following Ahmed glaucoma valve implantation and trabeculectomy with mitomycin-c, Korean I Ophthalmol, 2012;26:265-70

26. Costa VP, Correa MM, Kara-Jose N, Needling versus medical treatment in encapsulated blebs, Ophthalmology, 1997;104:1215-20

27. Giovingo $\mathrm{M}$, Complications of glaucoma drainage device surgery: a review, Semin Ophthalmol, 2014;29:397-402.

28. Kozobolis V, Konstantinidis A, Labiris G, Combined cataractglaucoma surgery. In: Rumelt S (ed), Glaucoma - Basic and Clinica Aspects. Rijeka: In Tech, 2013.

29. Singh K, Eid TE, Katz L, et al., Evaluation of Nd:YAG laser membranectomy in blocked tubes after glaucoma tube-shunt surgery, Am J Ophthalmol, 1997;124:781-6.

30. Olate-Pérez Á, Pérez-Torregrosa VT, Gargallo-Benedicto A, et al., Prospective study of filtering blebs after XEN45 surgery, Arch SOC Esp Oftalmol, 2017;92:366-71. 